\title{
Gaussian Process Inference for Estimating Pharmacokinetic Parameters of Dynamic Contrast-Enhanced MR Images
}

\author{
Shijun Wang ${ }^{1}$, Peter Liu ${ }^{1}$, Baris Turkbey ${ }^{2}$, Peter Choyke ${ }^{2}$, Peter Pinto ${ }^{2}$, \\ and Ronald M. Summers ${ }^{1}$ \\ ${ }^{1}$ Imaging Biomarkers and Computer-Aided Diagnosis Laboratory, Radiology \\ and Imaging Sciences, Clinical Center, National Institutes of Health, Bldg 10, \\ Room 1C224, Bethesda, MD 20892-1182, U.S. \\ \{wangshi, liupr\}@cc.nih.gov, rms@nih.gov \\ ${ }^{2}$ Molecular Imaging Program, National Cancer Institute, National Institutes of Health, \\ Bldg 10, Room B3B69F, Bethesda, MD 20892-1088, U.S. \\ \{Ismail_Turkbey, pchoyke, peter_pinto\}@nih.gov
}

\begin{abstract}
In this paper, we propose a new pharmacokinetic model for parameter estimation of dynamic contrast-enhanced (DCE) MRI by using Gaussian process inference. Our model is based on the Tofts dual-compartment model for the description of tracer kinetics and the observed time series from DCE-MRI is treated as a Gaussian stochastic process. The parameter estimation is done through a maximum likelihood approach and we propose a variant of the coordinate descent method to solve this likelihood maximization problem. The new model was shown to outperform a baseline method on simulated data. Parametric maps generated on prostate DCE data with the new model also provided better enhancement of tumors, lower intensity on false positives, and better boundary delineation when compared with the baseline method. New statistical parameter maps from the process model were also found to be informative, particularly when paired with the PK parameter maps.
\end{abstract}

Keywords: DCE-MRI, Gaussian Stochastic Process, Pharmacokinetic Model, Bayesian Inference, Coordinate Descent Optimization.

\section{$1 \quad$ Introduction}

Dynamic contrast-enhanced MR imaging (DCE-MRI) is a special magnetic resonance imaging (MRI) technique which assesses the micro-vascular status of tissue by repeated acquisition of rapid $\mathrm{T}_{1}$-weighted images on a region-of-interest (ROI) before, during and after the injection of a low molecular weight contrast agent $[1,2]$. The tracking of contrast agent, typically a gadolinium $(\mathrm{Gd})$ compound, provides a way to analyze the pharmacokinetics of the contrast agent which reveals information about the local vascular permeability, blood flow and extracellular volumes in the ROI.

The most widely used pharmacokinetic (PK) model in DCE is the Tofts' dualcompartment model [3], which models the exchange of contrast agent between the vascular space (blood plasma) and the extravascular-extracellular space (EES). The 
Tofts' model predicts for two quantitative parameters, $K_{\text {trans }}$ and $k_{e p}$, which have been shown to correlate with cancer ( $K_{\text {trans }}$ and $k_{e p}$ vary according to the organ being studied). These parameters are typically calculated by using nonlinear regression curvefitting techniques [4], which fit the concentration profiles of each MRI voxel independently, on a voxel-by-voxel basis. To challenge this voxel-independence assumption, Schmid et al. utilized adaptive Gaussian Markov random fields to estimate the kinetic parameters of DCE-MRI. In this analysis, neighboring voxels in the kinetic parameter estimation reduced the variability in local tumor regions and keep the boundaries between heterogeneous tissues sharp. This neighboring voxel approach was further developed with a spatial prior model by Kelm et al. [5].

Other probability models have also been used to treat the PK modeling problem. For example, Orton et al. utilize a full Bayesian approach [6]. Also, in the work of Chen et al., pixel-wise partial volume effect (PVE) was taken into account due to limited spatial resolution of DCE-MRI and tumor tissue heterogeneity [7]. However, in all of the probability models mentioned, it is assumed that the DCE-MRI observations at different time points are independent. This assumption does not hold for the real physical pharmacokinetic process because the observations from different time points have correlations imposed by the tracer dynamics across time. In reality, time dependence in DCE-MRI exists, and is difficult to characterize. In this paper, to capture the covariance between different time points of DCE-MRI, we treat the time series of DCE-MRI as a stochastic process and use a Gaussian process to describe it [8]. With the Gaussian process modeling, we estimated the pharmacokinetic and statistical parameters using maximum likelihood Bayesian inference.

The paper is organized as following: in Sec. 2, we first introduce the Tofts dualcompartment model briefly, and then propose a new method for the pharmacokinetic parameter estimation using Gaussian process inference (GPI); in Sec. 3 we show experimental results on both simulated data and the prostate MRI dataset; we conclude our findings in this study in Sec. 4 with a short discussion and future direction.

\section{Methods}

\subsection{Pharmacokinetic Model}

In this paper, we adopted a dual-compartment model proposed by Tofts et al. which was widely used to describe the dynamic uptake of contrast agent Gd-DTPA into the extracellular-extravascular space (EES) [3] (our method is a general framework and can also utilize other PK models). The concentration of Gd-DTPA as time goes by is modeled by the following equation:

$$
\mathrm{C}_{t}(x, t)=K^{\text {trans }}\left[C_{p}(x, t) \otimes \exp \left(-k_{e p} t\right)\right]
$$

where $\mathrm{C}_{t}(x, t)$ is the measured Gd-DTPA concentration in the tissue located at $x$ at time $t, C_{p}(x, t)$ is an arterial input function (AIF) giving the tracer concentration in blood plasma, $K_{\text {trans }}$ is the transfer constant between the blood plasma and EES, and $K_{e p}$ is the rate constant between the EES and blood plasma. The units of EES and blood plasma are both $\min ^{-1}$. $\otimes$ denotes the convolution operator. 
By using $\mathrm{T}_{1}$ weighted images, the Gd-DTPA concentration in tissue $\mathrm{C}_{t}(x, t)$ can be calculated from the following equation [9]:

$$
\mathrm{C}_{t}(x, t)=r_{1}^{-1}\left[T_{1}(x, t)^{-1}-T_{10}(x)^{-1}\right]
$$

where $T_{10}$ is the $T_{1}$ value before contrast agent administration, and $r_{1}=4.24 \mathrm{l} / \mathrm{s} / \mathrm{mmol}[3]$ is the longitudinal relaxivity of Gd-DTPA.

The Gd-DTPA concentration $C_{p}(x, t)$ in blood plasma is modeled by a biexponential function (arterial input function (AIF)) [3]:

$$
C_{p}(x, t)=D \sum_{i=1}^{2} a_{i} \exp \left(m_{i} t\right)
$$

where $a_{1}=3.99 \mathrm{~kg} / \mathrm{l}, \quad a_{1}=4.78 \mathrm{~kg} / l, \quad m_{1}=0.144 \mathrm{~min}^{-1}, m_{2}=0.0111 \mathrm{~min}^{-1}$ [10], and $D$ is the actual dose of contrast agent for a given body weight ( $D=0.1 \mathrm{mmol} / \mathrm{kg}$ in study [4]). In eq. (3) the first term models the short-term exchange of contrast agent with the tissues; the second term models the removal of contrast agent by the kidneys.

After substituting $C_{p}(x, t)$ in eq. (1) with eq. (3) and convoluting, we get:

$$
\mathrm{C}_{t}(x, t)=D K^{\text {trans }}\left(a_{1} \frac{\exp \left(-k_{e p} t\right)-\exp \left(-m_{1} t\right)}{m_{1}-k_{e p}}+a_{2} \frac{\exp \left(-k_{e p} t\right)-\exp \left(-m_{2} t\right)}{m_{2}-k_{e p}}\right)
$$

\subsection{Gaussian Process Inference}

Due to measurement noise in MR imaging and other sources of noise, the observation of tracer concentration in tissue on location $x$ at different time points $t=1,2, \ldots, T$ which is defined as $\mathbf{O}_{t}(x)=\left[\mathrm{O}_{t}(x, 1), \mathrm{O}_{t}(x, 2), \ldots, \mathrm{O}_{t}(x, T)\right]$ is a sequence of random variables, i.e., $\mathrm{O}_{t}\left(x, t_{i}\right)=C_{t}\left(x, t_{i}\right)+N\left(0, \sigma_{n}^{2}\right)$. We assume $\mathbf{O}_{t}(x)$ is a Gaussian stochastic process [8]:

$$
\mathbf{O}_{t}(x) \sim G P\left(\mathbf{C}_{t}(x), \boldsymbol{\Sigma}(x)\right),
$$

where $\mathbf{C}_{t}(x)=\left[\mathrm{C}_{t}(x, 1), \mathrm{C}_{t}(x, 2), \ldots, \mathrm{C}_{t}(x, T)\right]$ is the mean function and $\boldsymbol{\Sigma}(x)$ is the covariance function, $\Sigma_{i, j}\left(x, t_{i}, t_{j}\right)=\operatorname{cov}\left(\mathrm{C}_{t}\left(x, t_{i}\right), \mathrm{C}_{t}\left(x, t_{j}\right)\right)$.

Based on the observation that for DCE images, the images taken at time points which are close to each other on the time axis should have higher correlation than those taken at time points which are far away from each other on the time axis, we use the "squared exponential" covariance function [8], which fits the data well:

$$
\Sigma_{\mathrm{i}, j}\left(x, t_{i}, t_{j}\right)=\sigma_{f}^{2}(x) \exp \left[-\left(t_{j}-t_{i}\right)^{2} /\left(2 l(x)^{2}\right)\right]
$$


where $\sigma_{f}^{2}$ defines the maximum allowable covariance and $l$ defines the characteristic length-scale (the time required for a $\left[1-\mathrm{e}^{-0.5}\right]=39.4 \%$ decay in covariation).

By combining the covariance function shown in eq. (6) and the measurement noise with distribution $N\left(0, \sigma_{n}^{2}\right)$ from MR imaging together, we use the following covariance function:

$$
\Sigma_{\mathrm{i}, j}\left(x, t_{i}, t_{j}\right)=\sigma_{f}^{2}(x) \exp \left[-\left(t_{j}-t_{i}\right)^{2} /\left(2 l(x)^{2}\right)\right]+\sigma_{n}^{2}(x) \delta\left(t_{i}, t_{j}\right)
$$

where $\delta\left(t_{i}, t_{j}\right)$ is the Kronecker delta function. Zero mean Gaussian noise is used for simplicity, though non-zero mean Gaussian noise is easily implementable.

With the Gaussian process assumption, the log likelihood function is:

$$
\begin{aligned}
& L(x)=\log p\left(\mathbf{O}_{t}(x) \mid \mathbf{C}_{t}(x), K^{\text {trans }}, k_{e p}, \sigma_{f}, \sigma_{n}, l\right) \\
& =-\frac{1}{2} \log |\boldsymbol{\Sigma}(x)|-\frac{1}{2}\left(\mathbf{O}_{t}(x)-\mathbf{C}_{t}(x)\right)^{T} \boldsymbol{\Sigma}(x)^{-1}\left(\mathbf{O}_{t}(x)-\mathbf{C}_{t}(x)\right)-\frac{T}{2} \log (2 \pi)
\end{aligned}
$$

To maximize the likelihood function and find the values of hyperparameters $\left(K^{\text {trans }}, k_{e p}, \sigma_{f}, \sigma_{n}, l\right)$ introduced above, we use a variant of coordinate descent [11]; in our variation, the parameters are optimized in two groups: with $K_{\text {trans }}$ and $K_{e p}$ in one group, and $\sigma_{n}, \sigma_{f}$, and $l$ in the other. Formulas for the gradient follow:

$$
\begin{aligned}
& \frac{\partial L}{\partial K^{\text {trans }}}=\left(\mathbf{O}_{t}(x)-\mathbf{C}_{t}(x)\right)^{T} \boldsymbol{\Sigma}(x)^{-1} D\left[a_{1} \frac{\exp \left(-k_{e p} t_{i}\right)-\exp \left(-m_{1} t_{i}\right)}{m_{1}-k_{e p}}+a_{2} \frac{\exp \left(-k_{e p} t_{i}\right)-\exp \left(-m_{2} t_{i}\right)}{m_{2}-k_{e p}}\right]_{i=1, \ldots, T} \\
& \frac{\partial L}{\partial k_{e p}}=\left(\mathbf{O}_{t}(x)-\mathbf{C}_{t}(x)\right)^{T} \boldsymbol{\Sigma}(x)^{-1} D K^{\text {trans }} \times \\
& {\left[a_{1} \frac{\left(\exp \left(-k_{e p} t_{i}\right)-\exp \left(-m_{1} t_{i}\right)\right)-t_{i} \exp \left(-k_{\rho p} t_{i}\right)\left(m_{1}-k_{\varphi p}\right)}{\left(m_{1}-k_{c p}\right)^{2}}+a_{2} \frac{\left(\exp \left(-k_{e p} t_{i}\right)-\exp \left(-m_{2} t_{i}\right)\right)-t_{i} \exp \left(-k_{\varphi p} t_{i}\right)\left(m_{2}-k_{\varphi p}\right)}{\left(m_{2}-k_{c p}\right)^{2}}\right]_{i=1 . . T}} \\
& \frac{\partial L}{\partial \sigma_{f}^{2}(x)}=-\frac{1}{2} \operatorname{Tr}\left(\boldsymbol{\Sigma}(x)^{-T} \frac{\partial \boldsymbol{\Sigma}(x)}{\partial \sigma_{f}^{2}(x)}\right)+\frac{1}{2}\left(\mathbf{O}_{t}(x)-\mathbf{C}_{t}(x)\right)^{T} \times \boldsymbol{\Sigma}(x)^{-1} \frac{\partial \boldsymbol{\Sigma}(x)}{\partial \sigma_{f}^{2}(x)} \boldsymbol{\Sigma}(x)^{-1}\left(\mathbf{O}_{t}(x)-\mathbf{C}_{t}(x)\right) \\
& \frac{\partial \boldsymbol{\Sigma}_{i, j}\left(x, t_{i}, t_{j}\right)}{\partial \sigma_{f}^{2}(x)}=\exp \left[\frac{-\left(t_{j}-t_{i}\right)^{2}}{2 l(x)^{2}}\right] \\
& \frac{\partial L}{\partial l(x)}=-\frac{1}{2} \operatorname{Tr}\left(\boldsymbol{\Sigma}(x)^{-T} \frac{\partial \boldsymbol{\Sigma}(x)}{\partial l(x)}\right)+\frac{1}{2}\left(\mathbf{O}_{t}(x)-\mathbf{C}_{t}(x)\right)^{T} \times \boldsymbol{\Sigma}(x)^{-1} \frac{\partial \boldsymbol{\Sigma}(x)}{\partial l(x)} \boldsymbol{\Sigma}(x)^{-1}\left(\mathbf{O}_{t}(x)-\mathbf{C}_{t}(x)\right) \\
& \frac{\partial \boldsymbol{\Sigma}_{i, j}\left(x, t_{i}, t_{j}\right)}{\partial l(x)}=-2 \sigma_{f}^{2}(x) \exp \left[\frac{-\left(t_{j}-t_{i}\right)^{2}}{2 l(x)^{2}}\right] \times\left(-\frac{\left(t_{j}-t_{i}\right)^{2}}{2}\right) \times l(x)^{-3} \\
& \frac{\partial L}{\partial \sigma_{n}^{2}(x)}=-\frac{1}{2} \operatorname{Tr}\left(\boldsymbol{\Sigma}(x)^{-T} \frac{\partial \boldsymbol{\Sigma}(x)}{\partial \sigma_{n}^{2}(x)}\right)+\frac{1}{2}\left(\mathbf{O}_{t}(x)-\mathbf{C}_{t}(x)\right)^{T} \times \boldsymbol{\Sigma}(x)^{-1} \frac{\partial \boldsymbol{\Sigma}(x)}{\partial \sigma_{n}^{2}(x)} \boldsymbol{\Sigma}(x)^{-1}\left(\mathbf{O}_{t}(x)-\mathbf{C}_{t}(x)\right) \\
& \frac{\partial \boldsymbol{\Sigma}_{i, j}\left(x, t_{i}, t_{j}\right)}{\partial \sigma_{n}^{2}(x)}=\delta\left(t_{i}, t_{j}\right)
\end{aligned}
$$




\section{Experimental Results and Discussion}

The proposed Gaussian process inference (GPI) strategy was studied in two parts. First, the algorithm was tested on simulated data to assess its performance in estimating the "true" local pharmacokinetic parameters. Then, the algorithm was used to generate parametric maps using actual MRI-DCE axial scans of the prostate, and the resulting maps were qualitatively compared with histopathology results from radical prostatectomy after the DCE images were obtained. In both parts of the study, the GPI algorithm was compared against a standard least-squared-error (LSE) minimization method using a Nelder-Mead simplex algorithm. Squared-error-minimization is widely used in DCE-MRI [4].

\subsection{Results on Simulated Data}

For the simulated DCE data, we generated concentration profiles consisting of $46 \mathrm{Gd}-$ DTPA time-points spaced 5.6 seconds apart (spanning 4.3 minutes). The concentrations were assumed to follow the Gaussian probability distribution described in Sec. 2.2 with covariance terms $\sigma_{n}$ equaling $2 \%$ of the maximum Gd-DTPA concentration, $\sigma_{f}$ equaling $10 \%$ of the concentration peak, and $l$ equaling 30 seconds. Simulated data were generated for a physiologically relevant range of 0.1 to $1 \mathrm{~min}^{-1}$ for $K_{\text {trans }}$ and 0.2 to $3 \mathrm{~min}^{-1}$ for $K_{e p}$. Further, the constraint: $2 * K_{\text {trans }}<K_{e p}<10^{*} K_{\text {trans }}$ was imposed, allowing for $v_{e}$ (the EES fraction) to vary between 0.1 and 0.5 . For each $K_{\text {trans }} / K_{e p}$ pair, 100 independent random samples were generated.
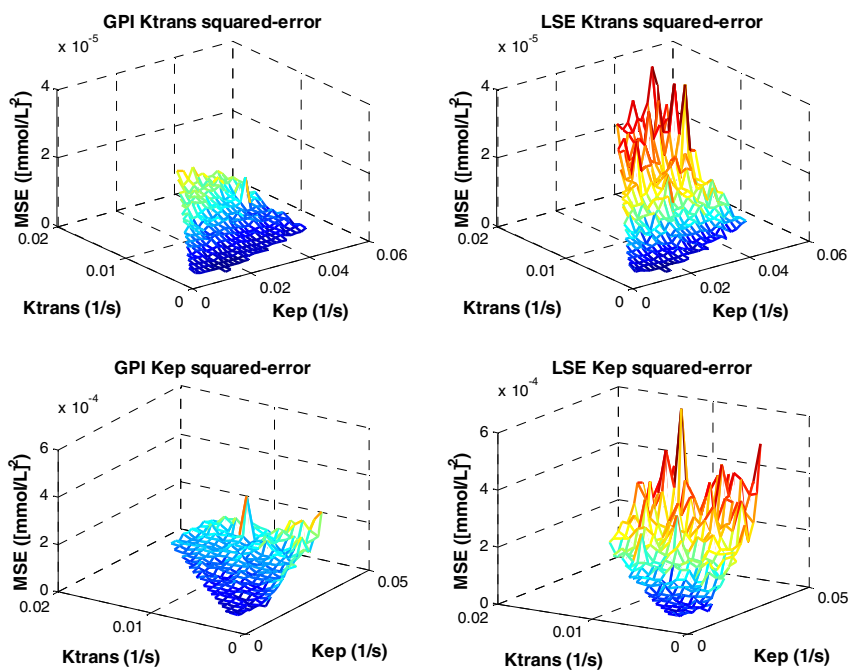

Fig. 1. Simulation results comparing the Mean Squared Errors from the GPI method (left) and the LSE method (right) in $K_{\text {trans }}$ (top) and $K_{e p}$ (bottom). On average, the GPI method resulted in lower errors than the LSE method ( $p<0.0001$ for both parameters). 
For each sample, the simulated DCE data was fit with both the GPI algorithm and the LSE algorithm. For each algorithm, the absolute error was calculated and averaged over all 100 samples (Figure 1). The results indicate that the GPI algorithm yields significantly lower squared errors than the LSE algorithm for multivariate Gaussian data with non-zero covariance (paired student $t$-test $p<0.0001$ for both $K_{\text {trans }}$ and $K_{e p}$ ).

\subsection{Results on Prostate MRI Data}

To test the clinical relevance of the GPI method, $K_{\text {trans }}$ and $K_{e p}$ maps were generated and compared using GPI and LSE methods. For this study, MR imaging studies of 26 patients were performed using a combination of an endorectal and cardiac coil on a 3T magnet (Philips Medical Systems). DCE-MRI and triplanar T2 weighted turbo-spin-echo

1)

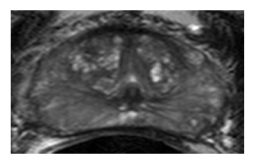

2)

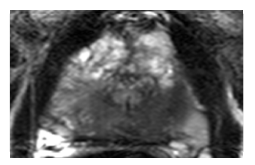

3)

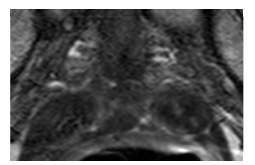

4)

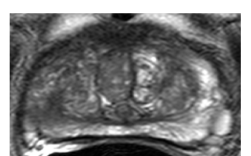

5)
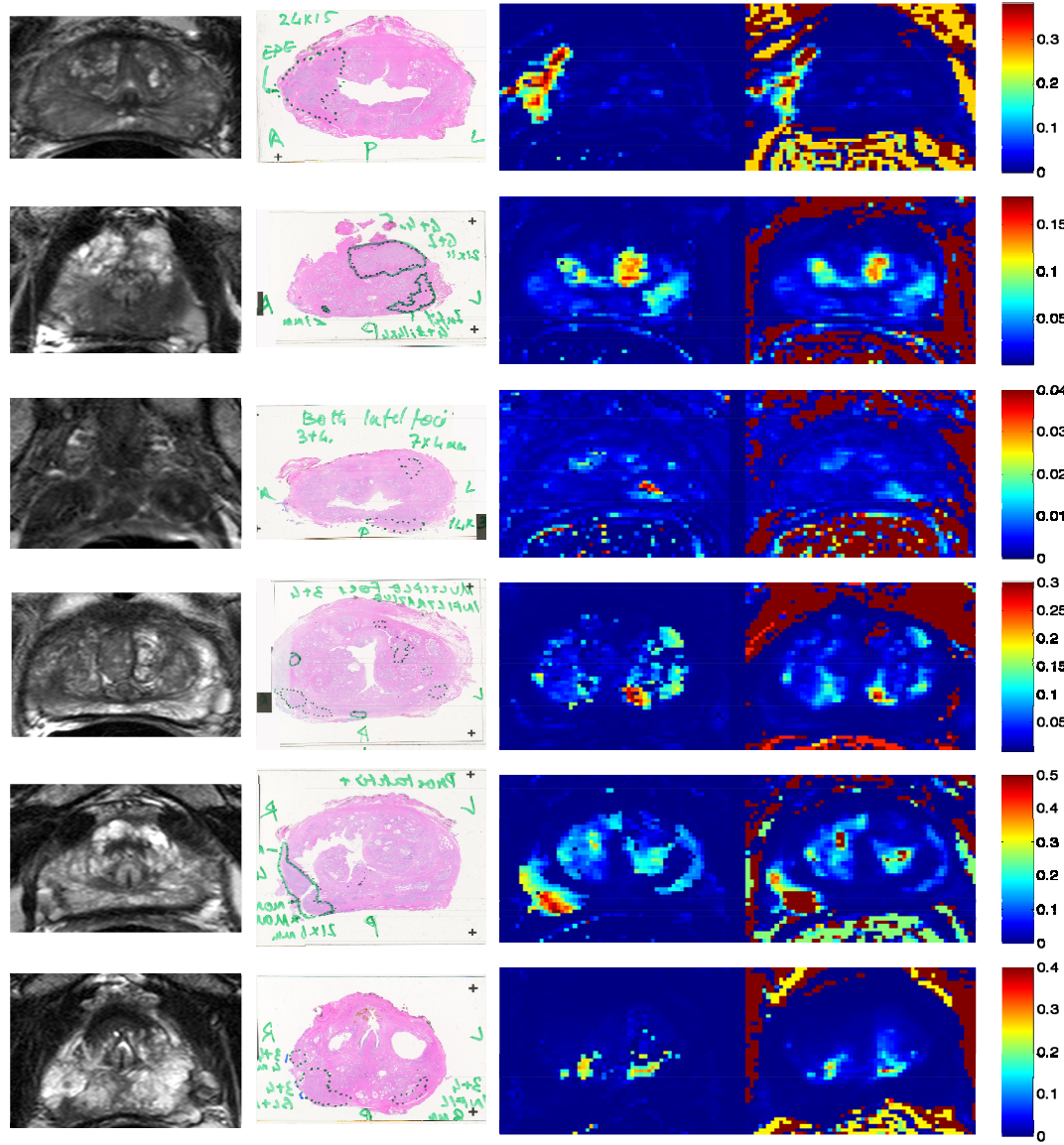

Fig. 2. GPI $K_{\text {trans }}$ maps (column 3) are compared with LSE $K_{\text {trans }}$ maps (column 4) for six patients. In column 2 , the corresponding histopathology labels for prostate cancer (inked in green) are shown. To better show the anatomical boundaries, registered T2-weighted MRI images are also shown (column 1). 
data sets were obtained. Following MRI, all patients underwent robotic radical prostatectomy. The prostate specimens were then sectioned within a customized mold system and mounted on a histopathology slide. Then, the tumors were outlined with their Gleason scores and dimensions in each prostatectomy specimen by two experienced genitourinary pathologists blinded to MRI data.

In Figure 2, parametric maps generated by GPI and LSE are shown next to ground truth histopathology cancer labels. Voxels where convergence was not met were excluded from the analysis. In the cases shown, the GPI maps show promise in increasing cancerous lesion enhancement. Particularly, in patients 1, 2, 3, 4 the cancerous regions indicated in the histopathology are more enhanced by GPI than LSE. Further, the GPI maps often reduce the enhancement of false positives shown to be benign. For example, in patient 5, the false-positives in the center of the prostate are darker in the GPI map than in the LSE map. In addition to these findings, it was also determined that the GPI maps tended to yield better outlines of the cancerous lesions. For example, patients $1,2,4,5$, and 6 all show a lesion shape in the GPI map that better coincides with the shape found in the histopathology data.

Another major advantage of our model over traditional models is that we can provide additional statistical parameter maps $\sigma_{n}, \sigma_{\mathrm{f}}$, and $l$ (Fig. 3). These maps reveal more information about tracer exchange kinetics in the tissue and provide complementary information to $K_{\text {trans }}$ and $K_{e p}$ maps. Particularly, it was found that the edges of prostate tumor often showed higher $\sigma_{\mathrm{f}}$ and $l$ values. This coincides with the finding that GPI provides better edge delineation of cancer lesions. For example, patient 1 in Fig. 2 showed a truer contour on the left and right edges of the lesion with GPI than LSE; this corresponds with the higher $\sigma_{f}$, and $l$ values exhibited in the same area in Fig. 3 (i.e. in areas where the covariance terms are high, GPI is expected to outperform LSE, as demonstrated in Fig. 1). The higher covariance at lesion boundaries is suspected to be caused by tissue heterogeneity.

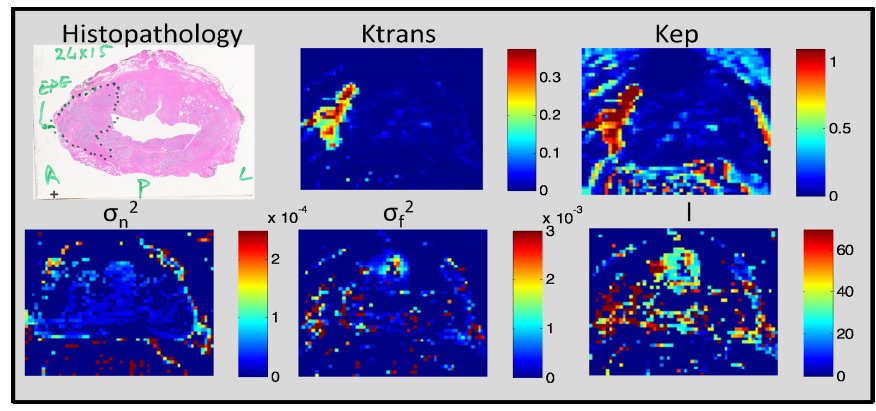

Fig. 3. All parametric maps generated by the GPI algorithm are shown with corresponding histopatholo-gy labels (cancer delineated in green). The color maps used for each parameter are show in the colorbar to the right of the image, with units in seconds ${ }^{-1}\left(K_{\text {trans }}\right.$ and $\left.K_{e p}\right), \mathrm{mmol} / \mathrm{L}$ $\left(\sigma_{n}^{2}\right.$ and $\left.\sigma_{f}^{2}\right)$, and seconds $(l)$.

\section{Conclusion}

In this paper, we propose a new pharmacokinetic model for parameter estimation of DCE-MRI by using Gaussian process inference. The parameter estimation is done 
through maximum likelihood approach and we propose a coordinate descent variant to solve this likelihood maximization problem.

By testing the algorithm on simulated MRI data, it was shown that the Gaussian process inference approach results in significantly more accurate results for MRI data that exhibits Gaussian covariance. Further, the $K_{\text {trans }}$ maps generated by the GPI algorithm show promise in yielding higher sensitivity and specificities, while leading to better delineations of lesion boundaries. $\sigma_{f}$, and $l$ also showed promise in aiding clinicians to detect tumor boundaries. In the future, quantitative comparisons of GPI with LSE and other PK methods are required to determine the true benefit of GPI.

Acknowledgments: The intramural research program of the NIH Clinical Center supported this work.

\section{References}

[1] Padhani, A.R., Leach, M.O.: Antivascular cancer treatments: functional assessments by dynamic contrast-enhanced magnetic resonance imaging. Abdominal Imaging 30, 324-341 (2005)

[2] O’Connor, J.P.B., Jackson, A., Parker, G.J.M., Jayson, G.C.: DCE-MRI biomarkers in the clinical evaluation of antiangiogenic and vascular disrupting agents. British Journal of Cancer 96, 189-195 (2007)

[3] Tofts, P.S.: Modeling tracer kinetics in dynamic Gd-DTPA MR imaging. JMRI-Journal of Magnetic Resonance Imaging 7, 91-101 (1997)

[4] Schmid, V.J., Whitcher, B., Padhani, A.R., Taylor, N.J., Yang, G.Z.: Bayesian methods for pharmacokinetic models in dynamic contrast-enhanced magnetic resonance imaging. IEEE Transactions on Medical Imaging 25, 1627-1636 (2006)

[5] Kelm, B.M., Menze, B.H., Nix, O., Zechmann, C.M., Hamprecht, F.A.: Estimating Kinetic Parameter Maps From Dynamic Contrast-Enhanced MRI Using Spatial Prior Knowledge. IEEE Transactions on Medical Imaging 28, 1534-1547 (2009)

[6] Orton, M.R., Collins, D.J., Walker-Samuel, S., d'Arcy, J.A., Hawkes, D.J., Atkinson, D., Leach, M.O.: Bayesian estimation of pharmacokinetic parameters for DCE-MRI with a robust treatment of enhancement onset time. Physics in Medicine and Biology 52, 2393-2408 (2007)

[7] Chen, L., Choyke, P.L., Chan, T.H., Chi, C.Y., Wang, G., Wang, Y.: Tissue-Specific Compartmental Analysis for Dynamic Contrast-Enhanced MR Imaging of Complex Tumors. IEEE Transactions on Medical Imaging 30, 2044-2058 (2011)

[8] Rasmussen, C., Williams, C.: Gaussian Processes for Machine Learning. MIT Press (2006)

[9] Buckley, D., Parker, G.: Measuring contrast agent concentration in T1-weighted dynamic contrast-enhanced MRI. Presented at Dynamic Contrast-Enhanced Magnetic Resonance Imaging in Oncology (2005)

[10] Rohrer, M., Bauer, H., Mintorovitch, J., Requardt, M., Weinmann, H.J.: Comparison of magnetic properties of MRI contrast media solutions at different magnetic field strengths. Investigative Radiology 40, 715-724 (2005)

[11] Bouman, C.A., Sauer, K.: A unified approach to statistical tomography using coordinate descent optimization. IEEE Transactions on Image Processing 5, 480-492 (1996) 